\title{
Design, Optimization, Calibration, and a Case Study of a 3D-Printed, Low-cost Fingertip Sensor for Robotic Manipulation
}

\author{
Zhe Xu, Svetoslav Kolev and Emanuel Todorov
}

\begin{abstract}
We describe a low-cost 3-axis fingertip force sensor for robotic manipulation. Our design makes the most of 3D printing technology, and takes important factors such as maintainability and modification into consideration. The resulting sensor features a detachable fingertip made of 3Dprinted materials, and a cantilever mechanism that allows the detection of contact forces via three off-the-shelf, low-cost force sensors. To improve our design concept, optimization on the configuration of the fingertip sensor is performed under statistical analysis of the hysteresis performance. The optimized fingertip sensor is experimentally investigated and calibrated. At the end, through a case-study, we demonstrate that our proposed design can measure the direction of contact forces in the radial plane of the fingertip sensor.
\end{abstract}

\section{INTRODUCTION}

During manipulation and exploration tasks, robotic hands/grippers are often required to respond effectively to unknown objects and obstacles. Without detailed models of the operating environment, real-time measurement of the contact force is essential for the success of a robotic hand/gripper to perform dexterous tasks. As thoroughly summarized in a recent review [1], many different types of force/torque sensors have been developed over the past decade in order to equip the robotic hands with the tactile sensing ability in various applications.

When it comes to choosing the suitable fingertip sensors for a robotic hand/gripper, besides taking into consideration the sensing ability, the cost still plays an important role. So far, high-end fingertip sensors have been widely employed by many advanced robotic hands [2]-[5]. However, the cost of those sensors accounts for large portions of the robotic hand. The price of a commercially available 6-axis force/torque sensor starts at $\$ 2000$. Although the performance of those advanced tactile/force-torque sensors are very appealing, there also exist situations where the researchers have to comply with their limited budgets on fingertip sensors and would like to reasonably relax the sensing requirement.

Durability and maintainability are the other two parameters that were often ignored during the design of fingertip sensors. The skin of the human hand can regenerate itself, and therefore normal wear and tear won't cause permanent damages to sensing modalities. The increasing roles of robots in human environments require robotic hands to be operated in harsh conditions where a fingertip sensor may be exposed to corrosive chemicals, metal objects with sharp edges, and

Authors are with the Department of Computer Science \& Engineering, University of Washington, WA 98195, USA

e-mail: zhexu@cs.washington.edu, swetko@cs.washington.edu, todorov@cs.washington.edu coarse surfaces of certain objects that can all cause severe damages to the fingertip sensors beyond the normal repair. However, most of existing fingertip sensors have either embedded sensing elements [6] at the contact region or layered flexible circuit [2], [7] under the elastic materials of fingertips. These features can potentially raise the risk of broken sensors. Therefore, important factors such as lowcost, maintainability and durability have to be taken into consideration during the design of future fingertip sensors.

In recent years, many researchers have started using 3Dprinted parts directly for their robots, or sometimes fabricating the entire working prototype using only 3D printed parts with a few fasteners [8], [9]. 3D-printed molds and frames were also developed for making different soft skin textures [7], [10], [11]. Therefore, it is of our interest to investigate the possibility of designing and fabricating a 3D-printed fingertip sensor, and to understand essential characteristics that may potentially affect the performance of fingertip sensors.

To this end, we describe a fingertip sensor that makes the most of the appealing features of low-cost force sensors, innovative mechanism, and 3D-printing technology. In contrast to conventional approaches, our proposed design moves the sensing components away from the contacting areas to the base of the fingertip and keeps only the low-cost, compliant fingertip for direct contact with objects. These changes can potentially reduce the cost, allow more task-oriented design, and make the maintenance of the fingertip sensors easier than using complicated/expensive sensors.

In the following sections, the design concept of the fingertip sensor is explained, the important components are detailed, and then we optimize the configurations of our proposed fingertip sensor based on the hysteresis analysis. An optimized fingertip sensor is then used for calibration. At the end, based on what we learn from the 3D-printed fingertip sensor, we demonstrate a case study of our proposed design adapted to a robotic manipulation task with little modification efforts.

\section{DeVElopment OF THE FINGERTIP SENSOR}

This section describes the working principle, the mechanical structure, and the electronic components of our proposed fingertip sensor.

\section{A. Sensor description and the working principle}

As shown in Fig. 1, the working principle of the fingertip sensor is based on a cantilever mechanism. The fixed end is located at the bottom of the base; the free end is attached 

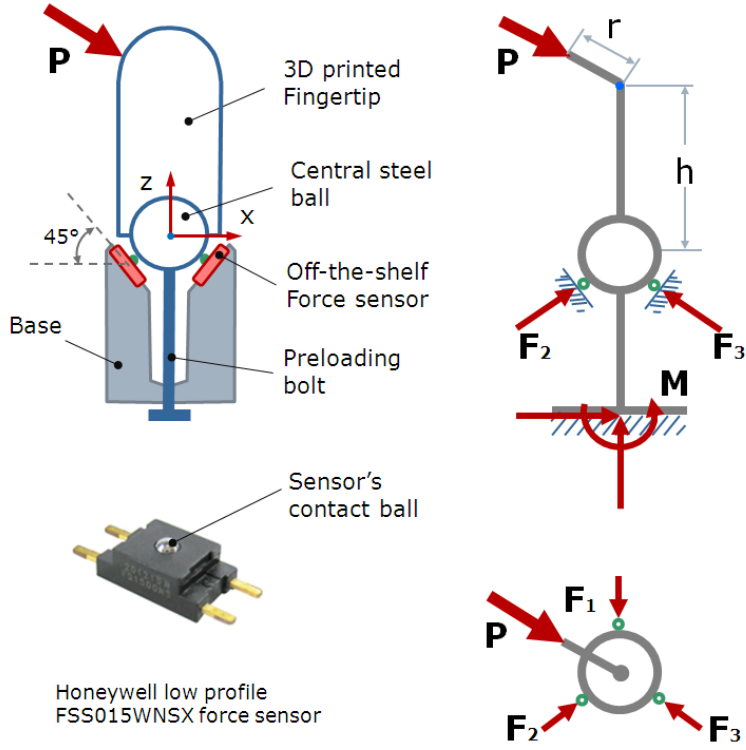

Fig. 1. Schematic drawing and force analysis of our proposed fingertip sensor. Top left: Schematic drawing of the fingertip sensor showing relative positions of all components. Bottom left: The picture of a Honeywell force sensor used in this paper. Right column: Beam structure of the fingertip sensor when external force $(\mathrm{P})$ applied at the fingertip.

to the bottom of the fingertip through a central steel ball. The central steel ball is then supported by three symmetrically arranged Honeywell FSS015WNSX force sensors (FSS sensor hereinafter) via three pairs of sphere contacts. The three FSS sensors are placed at the inclined opening of the well structure of the base. The external forces applied at the fingertip will result in a combined reaction force from the three sensor balls and get detected by three FSS sensors.

As represented as a beam structure in Fig. 1, important parameters that are related to manipulation task contains the radius $(r)$ and the height $(h)$ of the fingertip. The diameter of the central steel ball decides the size of the base structure which is designed to not have contact with external objects, and therefore won't be considered as a design parameter. Additionally, because the exposed surfaces of the central steel ball and three sensor balls are all plated, the contact between them can be retreated as frictionless sphere contacts, thus the sensor reading should mainly reflect the change from the normal forces $\left(F_{1}, F_{2}\right.$, and $\left.F_{3}\right)$ at the contact sites.

\section{B. Mechanical design of the fingertip sensor}

As shown in Fig. 2, our proposed fingertip sensor is composed of five components, namely, a fingertip (can be coated with silicone rubber), a plated central steel ball, a force sensor enclosed base, a cantilever-bolt mounted bottom cap, and three FSS force sensors. Except for the extended cantilever-bolt (a M2.5 steel screw), the central steel ball (9.4 $\mathrm{mm}$ in diameter) and the FSS sensors, all the other parts were 3D printed by the Dimension BST 768 (Stratasys Corp., Eden Prairie, MN) within one hour.

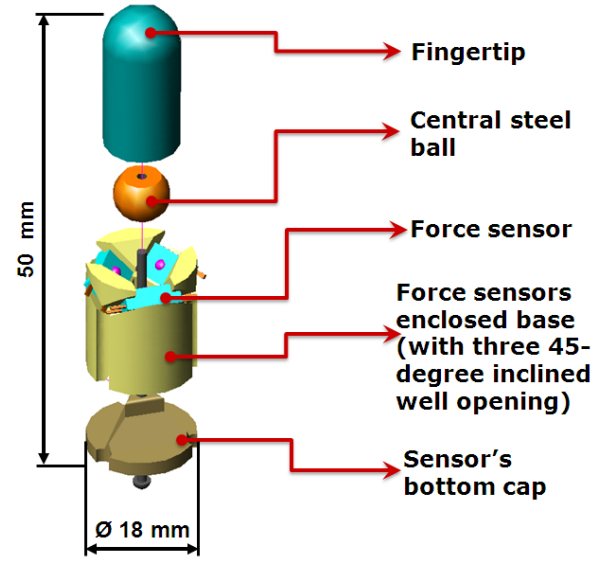

Fig. 2. 3D CAD model of the fingertip sensor.

\section{Electrical wiring}

The Honeywell FSS sensor is powered by 5V DC source. Its output is then fed into an instrumentation amplifier LT1920 from Linear Technologies, where the signal is differentially amplified by a factor of 5 . The output is fed to a NI-DAQ PCI 6229 card where it is digitized as shown in Fig. 3. And the detail specifications of the FSS sensor are listed in Table I.

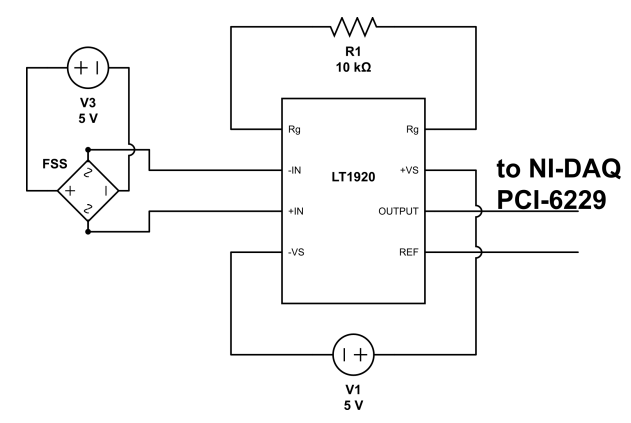

Fig. 3. The electrical wiring diagram of a single FSS sensor.

TABLE I

PerformanCE CHARACTERISTICS OF THE FSS FORCE SENSOR

\begin{tabular}{rrllll}
\hline & & \multicolumn{3}{c}{ sensor FSS015WNSX } \\
\cline { 4 - 6 } Characteristic & Unit & Min. & Typ. & Max. \\
\hline Force sensing range(FS & $\mathrm{N}$ & 0 & - & 15 \\
Operating voltage & $\mathrm{Vdc}$ & 3.3 & 10 & 12.5 \\
Zero offset & $\mathrm{mV}$ & -30 & 0 & +30 \\
Zero shift & & & & \\
Linearity & $\% \mathrm{span}$ & - & \pm 0.5 & - \\
Sensitivity & $\mathrm{mV} / \mathrm{V} / \mathrm{N}$ & 2.2 & 2.4 & - \\
Repeatability & $\% \mathrm{span}$ & - & \pm 0.2 & - \\
Response time & & & & \\
$(10 \%$ to $90 \% \mathrm{FS})$ & $\mathrm{ms}$ & - & 0.1 & 0.5 \\
Safety overforce & $\mathrm{N}$ & - & - & 45 \\
\hline
\end{tabular}




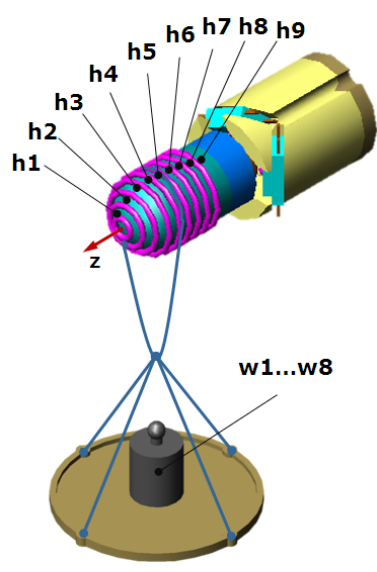

(a) Experimental group 1(Gl)

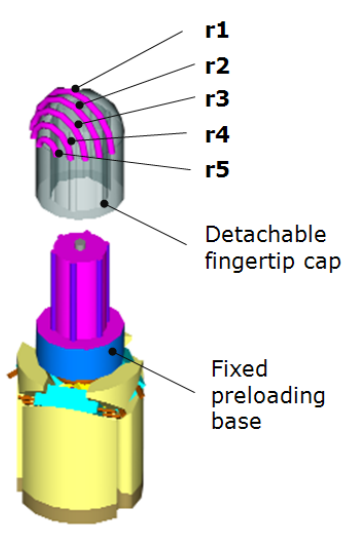

(b) Experimental group 2(G2)

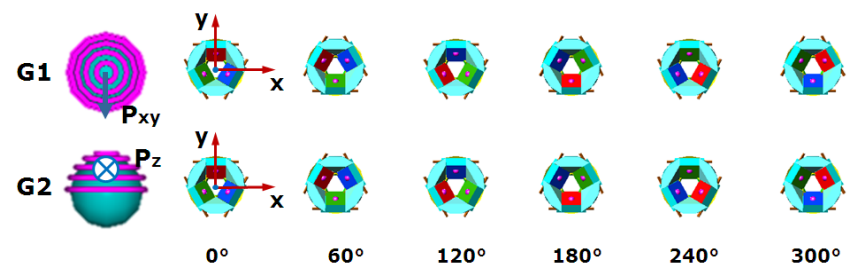

(c) The same fingertip sensor was tested with a set of 8 accumulative weight with two different orientations (G1 and G2) under six different rotational conditions, respectively.

Fig. 4. Varing conditions for design optimization. (a) Detachable fingertip cap for minimizing the disturbance to the pre-loading status. (b) Hanging weight method used for loading/unloading difference forces. (c) Under the same force conditions, six different rotational cases were tested during the experiments. (Note: the red, green, and blue colors were used to label the three FSS sensors.)

\section{DESIGN OPTIMIZATION OF THE FINGERTIP SENSOR}

According to the factory's specification, the performance of the Honeywell FSS sensor is very good (as detailed in Table I), and therefore they are often adopted by medical devices [12]. However, due to the special structure we adopted for our design, once the three FSS sensors are installed inside the fingertip sensor, the central steel ball would exert compression force through the three contacting balls of the FSS sensors. It is of interest to understand how the FSS sensors behave under pre-loading. Besides, the overall performance of our proposed fingertip sensor relies heavily on its mechanical design. Important factors such as the friction between the central steel ball and the three sensor's contacting balls, and the elastic behavior of the ABS plastic used for 3D printing can all be greatly affected by the configurations of the fingertip sensor. In order to investigate how the mechanical design will affect the performance of our proposed fingertip sensor, we conducted hysteresis analysis with respective to two groups of forces (G1-type and $G 2$ type) under six different rotational conditions as illustrated in Fig. 4.

At the beginning of each loading test [13], a small tray was hanged from the fingertip (at $h 1 \ldots h 9$ or $r 1 \ldots r 5$ ) through a pair of strings. The tray and strings weigh 5 grams in total. During the experiments, known weights were added
TABLE II

SPECIFICATIONS OF THE TESTED FINGERTIP FORCE SENSOR

\begin{tabular}{rrl}
\hline Term & Unit & Value \\
\hline Tested height $\left(h_{1} \ldots h_{9}\right)$ & $\mathrm{mm}$ & 22.7621 .9220 .5218 .72 \\
& & 16.7614 .7612 .7610 .768 .76 \\
Tested radius $\left(r_{1} \ldots r_{5}\right)$ & $\mathrm{mm}$ & 01.903 .695 .126 .03 \\
Hanging weights $\left(w_{1} \ldots w_{8}\right)$ & $\mathrm{gram}$ & 05153555105132232 \\
Total weight & gram & 18 \\
Total height & $\mathrm{mm}$ & 48.50 \\
Total diameter & $\mathrm{mm}$ & 18.00 \\
FSS sensor's price & \$/unit & 60 \\
\hline & &
\end{tabular}

and removed from the tray manually, and force readings from the FSS sensors were recorded by NI DAQ PCI-6229 at $2 \mathrm{KHz}$. In order to obtain the hysteresis curves we initialized the fingertip sensor with the empty tray. We then successively added weights to the tray so that we obtained measurements at different weights. After that we removed the weights in the reverse order. After each addition/removal we waited until the weight stabilized and marked the time in our recording with the corresponding weight. We then averaged the values from the 3 seconds after the time marker and treated that number as the voltage output of the sensor under the loaded weight.

Group 1 and group 2 each have distinctively nine and five testing locations. And for every testing location, loading and uploading experiments were conducted with eight different weights. The information of the tested fingertip sensor is listed in Table II. Examples of processed data are as shown in Fig. 5. Due to the small non-linearity effects found from the data, we chose to use the following, simplified equation to calculate the midpoint hysteresis for every testing location:

$$
\text { Hysteresis } \%=\left|\frac{S_{m p}-S_{m n}}{S_{\text {start }}-S_{\text {end }}}\right| \times 100 \%
$$

where $S_{\text {start }}$ and $S_{\text {end }}$ are the sensor readings from the start and end points of the hysteresis loop; $S_{m p}$ and $S_{m n}$ are the readings in a positive and negative going directions, respectively. Based on Eq. (1), the mechanical hysteresis of the fingertip sensor was statistically analyzed as shown in Fig. 6.

In both group 1 and 2's experiments, a cantilever effect is formed when the external forces $\left(P_{x y}\right.$ or $\left.P_{z}\right)$ are applied at the fingertip (the free end) of our 3D-printed force sensor. The readings of the three FSS sensors located at the base (the fixed end) will increase if they are on the compression side, or decrease if they are on the tension side.

A typical loading/unloading process is shown in Fig. 5(a) for group 1's data. An exponential decrease of the hysteresis with the height of the fingertip was observed. This can be resulted from the decreasing torque effect on the mechanical hysteresis as the loading/unloading forces moving towards the based of the fingertip sensor. When the external force $P$ is applied according to the group 1's setup, in the x-y plane of the fingertip sensor, torques are simultaneously generated at the contact sites between the central steel ball and three 

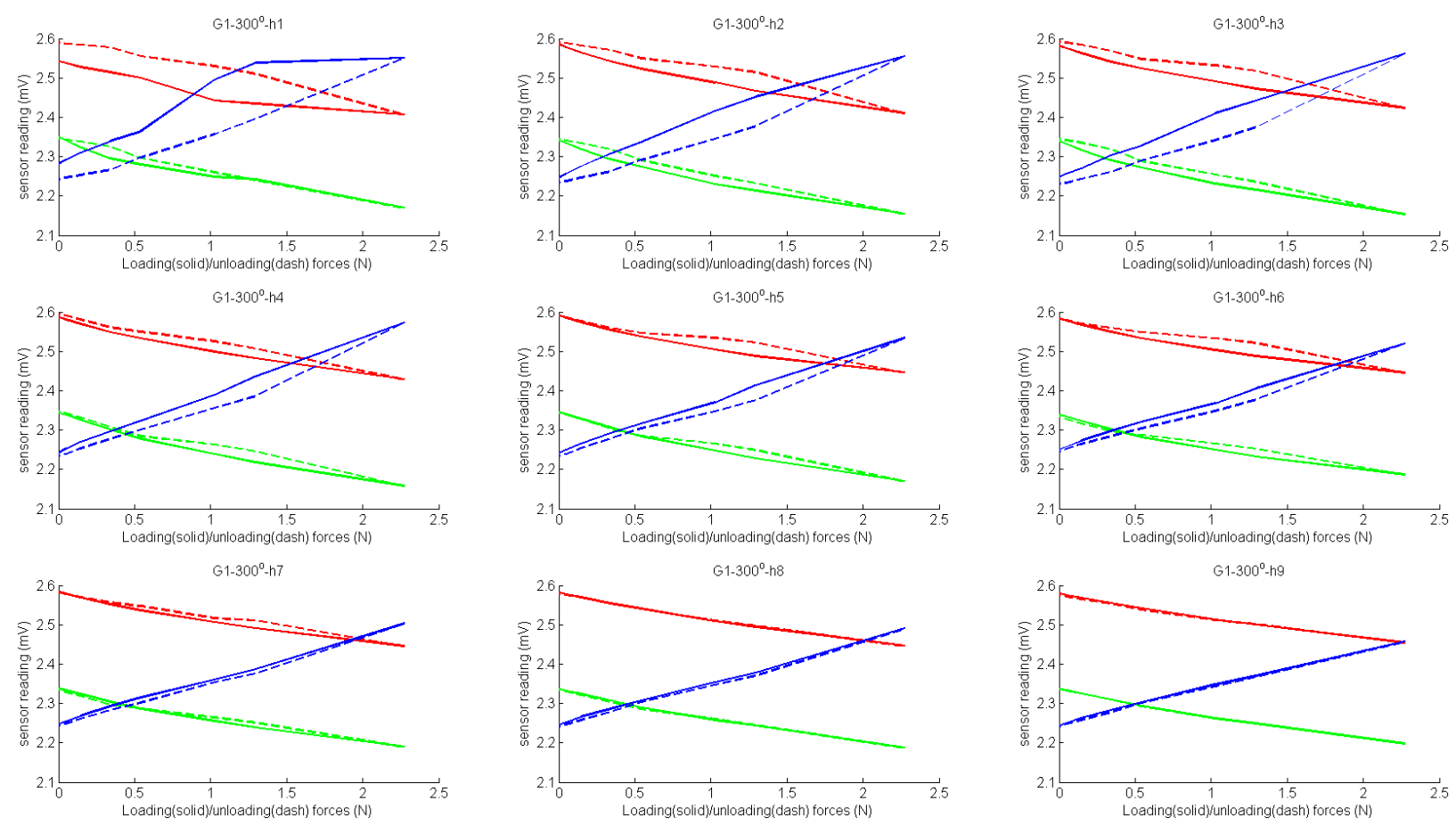

(a) A set of $P_{x y}\left(8\right.$ accumulative weights) applied at 9 different axial locations when the rotation-angle is fixed at $300^{\circ}$ for G1.
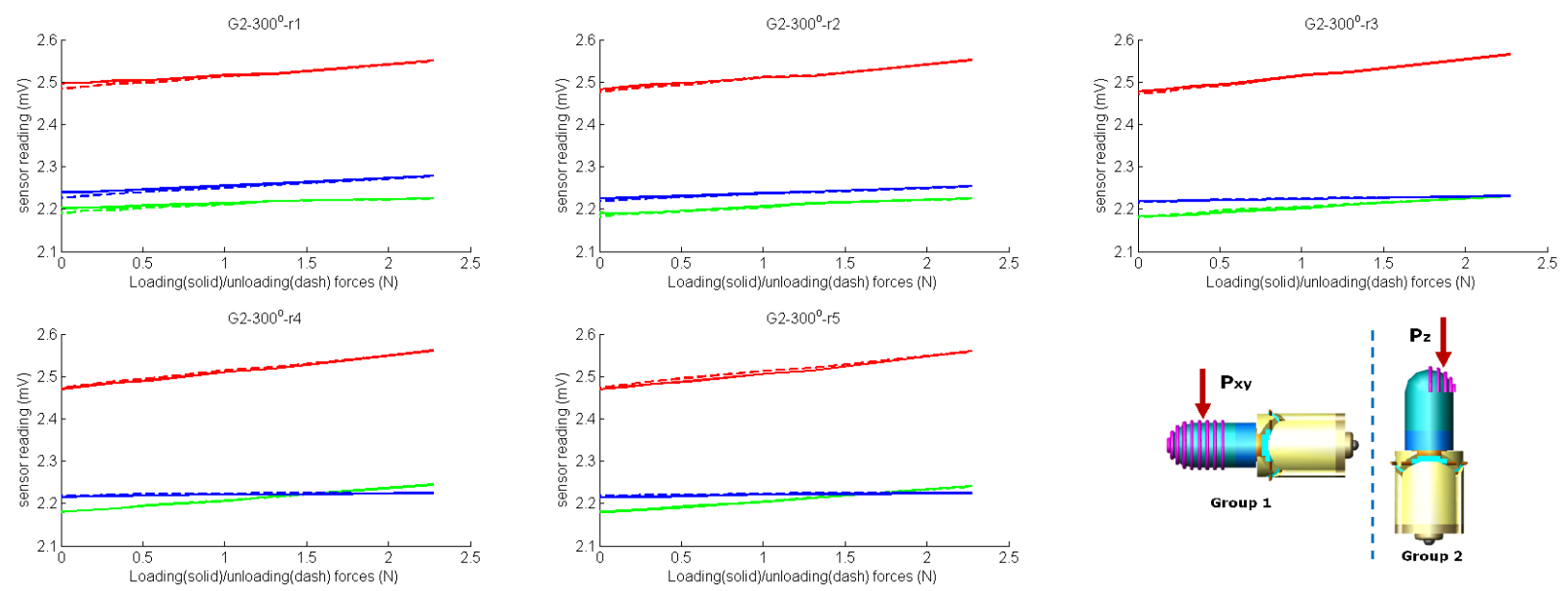

(b) A set of $P_{z}\left(8\right.$ accumulative weights) applied at 5 different radial locations when rotation-angle is fixed at $300^{\circ}$ for $\mathrm{G} 2$.

Fig. 5. Examples of the data collected for hysteresis analysis. (Note: the red, green, and blue data points were collected from the three sensors defined in Fig. 4(c), respectively.)

FSS sensors' contacting balls. Although the friction between two plated metal balls is seemly small compared to the force applied to the fingertip, the induced friction could still cause the reading of a compressed FSS sensor (see the blue curves in Fig. 5(a)) to increase from 2.46 to $2.56 \mathrm{mV}$ under the same maximum loading force, accounting for a $33.9 \%$ of the total increasing force from 0 to $2.27 \mathrm{~N}$, as shown in Fig. 5(a) from moving the test location from $h 9$ to $h 1$.

Interestingly, a similar but smaller, 9.6\% increase of the sensor reading under the maximum compression force was observed in group 2's data (see the red curve in Fig. 5(b)) as the z-direction forces $P_{z}$ moving from $r 1$ to $r 5$. This is because the change of moment arm decreases by two times when the same amount of testing forces are moved from group 1 to group 2 .

On the contrary, the lowest, averaged hysteresis for group 2's data (2.19\%) still appears to be over two times larger than the lowest one from group 1's data $(0.98 \%)$ (see Fig. 6). This is because the total span of the sensor readings (the denominator $S_{\text {start }}-S_{\text {end }}$ in Eq. (1)) in group 2 becomes very small (about $0.005 \mathrm{mV}$ ) for the sensors on the tension side of the fingertip during the six different rotations (see blue curve in Fig. 5(b)).

Based on the above analysis, we found that the height of the 3D-printed fingertip sensor will affect the hysteresis performance of the sensor most, therefore we redesigned an 


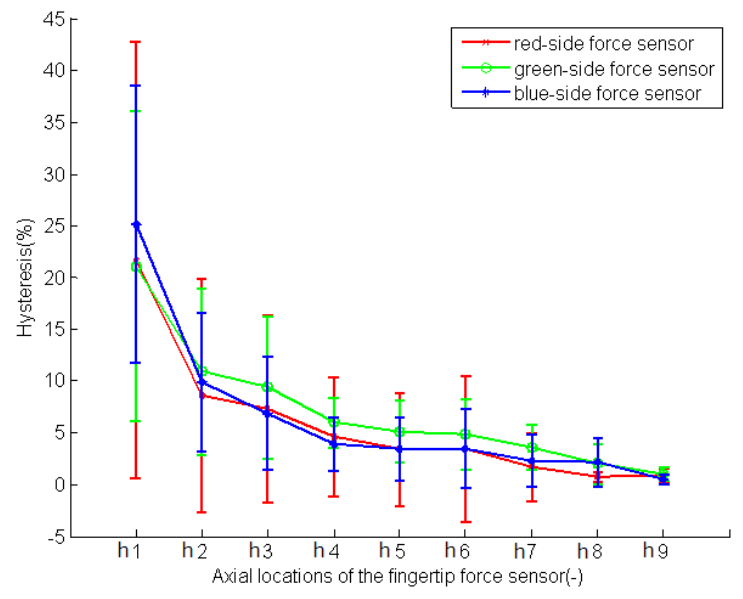

(a) The hysteresis along axial direction of the finger force sen$\operatorname{sor}(G 1)$

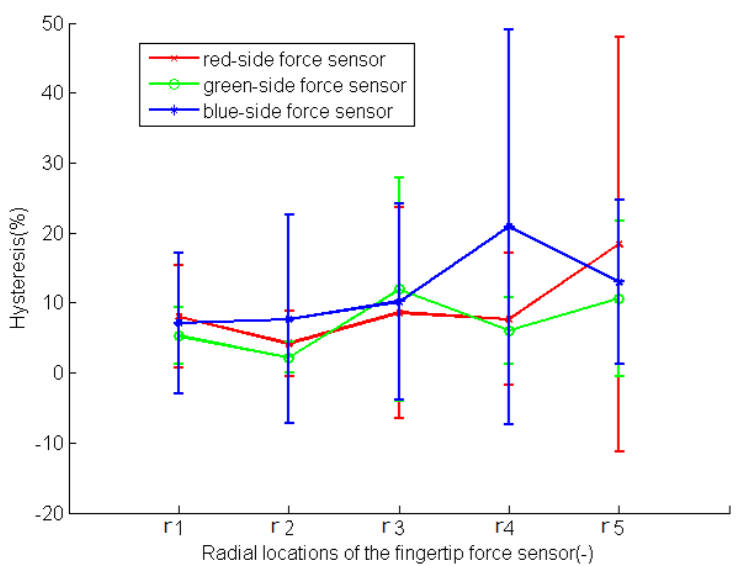

(b) The hysteresis along radial direction of the finger force sensor $(G 2)$

Fig. 6. The change of the hysteresis of the proposed fingertip sensor at different testing locations.

TABLE III

SPECIFICATIONS OF THE OPTIMIZED FINGERTIP FORCE SENSOR

\begin{tabular}{rrll}
\hline Term & Unit & Value \\
\hline Tested height $\left(h_{1} \ldots h_{6}\right)$ & $\mathrm{mm}$ & 8.417 .716 .625 .193 .511 .65 \\
Tested radius $\left(r_{1} \ldots r_{6}\right)$ & $\mathrm{mm}$ & 1.653 .515 .196 .627 .718 .41 \\
Hanging weights $\left(w_{1} \ldots w_{10}\right)$ & gram & 0515355582109136163213 \\
Total weight & gram & 14 & \\
Total height & $\mathrm{mm}$ & 20 & \\
Total diameter & $\mathrm{mm}$ & 18.00 & \\
\hline
\end{tabular}

optimized fingertip force sensor that has a height $(h)$ of $0 \mathrm{~mm}$ and a radius $(r)$ of $9 \mathrm{~mm}$ based on the schematic drawing in Fig. 1. The calibration of the optimized force sensor will be detailed in the following section.

\section{CAlibRation OF AN OPTIMIZED FINGERTIP SENSOR}

The specifications of our optimized fingertip sensor is summarized in Table III.

Before the calibration, a frictionless hard-finger model is chosen for the force analysis of the optimized fingertip sensor as shown in Fig. 7. When there is an external force applied
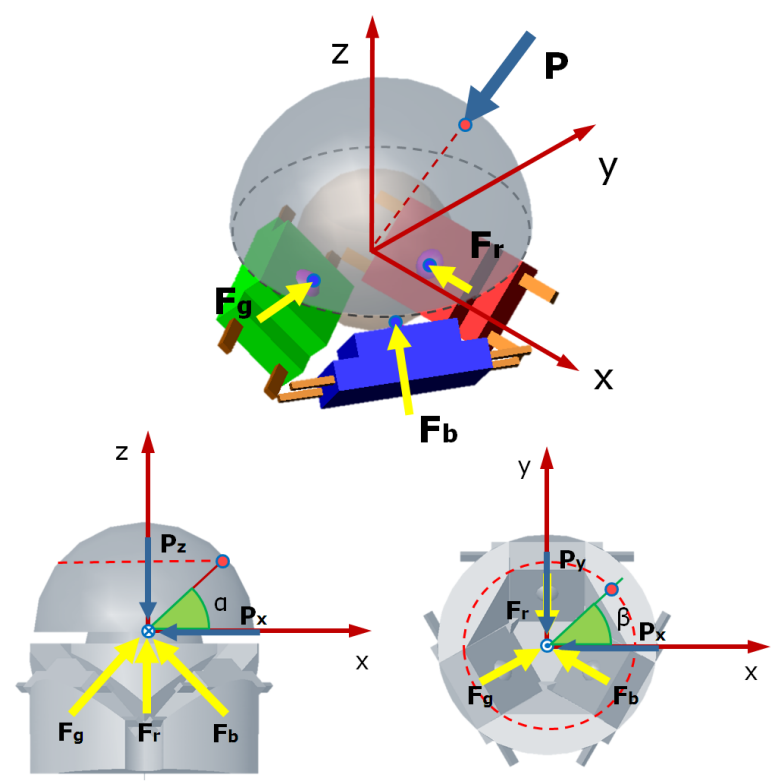

Fig. 7. Force analysis of the fingertip force sensor.

on the surface of fingertip, its normal component $(\vec{P})$ can be further decomposed into three force vectors $\left(\vec{P}_{x}, \vec{P}_{y}\right.$, and $\left.\vec{P}_{z}\right)$ in the fingertip's coordinate system. For the fingertip at equilibrium, three counteracting forces $\left(\vec{F}_{r}, \vec{F}_{g}\right.$, and $\left.\vec{F}_{b}\right)$ will be transmitted simultaneously through the three FSS sensor's contacting balls. Since we have already reduced the height of the fingertip after the optimization, the torque induced friction is neglected at the contact point between the central ball and the three FSS sensors. And the following force balance can be achieved:

$$
\vec{P}=\vec{F}_{r}+\vec{F}_{g}+\vec{F}_{b}
$$

where the normal force component $(\vec{P})$ can be further decomposed with location information $\alpha$ and $\beta$ :

$$
\left(\begin{array}{c}
\overrightarrow{P_{x}} \\
\overrightarrow{P_{y}} \\
\overrightarrow{P_{z}}
\end{array}\right)=\left(\begin{array}{c}
\vec{P} \cdot \cos \alpha \cdot \cos \beta \\
\vec{P} \cdot \cos \alpha \cdot \sin \beta \\
\vec{P} \cdot \sin \alpha
\end{array}\right)
$$

From the 3D model of the fingertip sensor, the geometric information containing the locations of the three FSS sensors is used to formulate the counteracting forces:

$$
\begin{gathered}
\vec{F}_{r}=\left(0,-\cos 45^{\circ}, \sin 45^{\circ}\right)^{T} \cdot k_{r} \cdot S_{r} \\
\vec{F}_{g}=\left(\cos 45^{o} \cos 30^{\circ}, \cos 45^{\circ} \sin 30^{\circ}, \sin 45^{\circ}\right)^{T} \cdot k_{g} \cdot S_{g} \\
\vec{F}_{b}=\left(-\cos 45^{\circ} \cos 30^{o}, \cos 45^{o} \sin 30^{\circ}, \sin 45^{o}\right)^{T} \cdot k_{b} \cdot S_{b}
\end{gathered}
$$

Where $k_{r}, k_{g}, k_{b}$, and $S_{r}, S_{g}, S_{b}$ are the calibration coefficients and the sensor readings from the correspondingly colored FSS sensors (see Fig. 7), respectively.

Then by substituting Eq. (3)-(6) into Eq. (2), we derive the fingertip model as follows: 


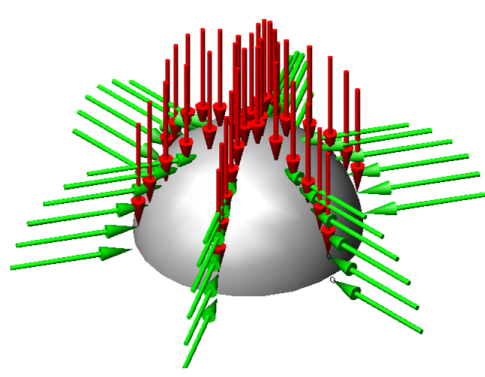

Fig. 8. 72 sets of loading forces applied at different locations on an optimized fingertip sensor for calibration purpose. Note: $G 1$ and $G 2$-type forces are labeled with green and red colors, respectively.

$$
\left(\begin{array}{c}
\overrightarrow{P_{x}} \\
\overrightarrow{P_{y}} \\
\overrightarrow{P_{z}}
\end{array}\right)=\boldsymbol{A} \cdot\left(\begin{array}{c}
S_{r} \\
S_{g} \\
S_{b}
\end{array}\right)
$$

where coefficient matrix $A$ is in the form of:

$$
\boldsymbol{A}=\left(\begin{array}{ccc}
0 & 0.6124 & -0.6124 \\
-0.7071 & 0.3536 & 0.3536 \\
0.7071 & 0.7071 & 0.7071
\end{array}\right) \cdot\left(\begin{array}{ccc}
k_{r} & 0 & 0 \\
0 & k_{g} & 0 \\
0 & 0 & k_{b}
\end{array}\right)
$$

As shown in Fig. 8, a total of 72 sets of loading forces were applied at 36 different locations ${ }^{1}$ on the optimized fingertip sensor in two groups. The normal component of forces $(\vec{P})$ together with their acting locations $(\alpha$ and $\beta)$ were extracted for the calibration process.

The calibration matrix was computed by using least square optimization method from Matlab. For the optimized fingertip sensor, a full rank coefficient matrix $\boldsymbol{A}_{\text {opt }}$ suggests that three force components of the external force $(\vec{P})$ can be independently decomposed. And the condition number of $\boldsymbol{A}_{\text {opt }}$ is 34.92 .

$$
\boldsymbol{A}_{\text {opt }}=\left(\begin{array}{ccc}
-0.1569 & -2.7179 & 0.0155 \\
2.3755 & 1.4390 & 0.1033 \\
-2.1593 & 1.3978 & 0.0545
\end{array}\right)
$$

The comparison between applied and estimated forces is shown in Fig. 9. It is interesting to find that our proposed fingertip sensor is able to estimate the rotation of the external force in the $\mathrm{x}-\mathrm{y}$ plane quite well, but has difficulties of measuring the force $\left(\vec{P}_{z}\right)$ in axial direction. One possibility is that the direction of the $\vec{P}_{z}$ is coincident with, but opposite to the direction of the pre-loading force, thus the bigger the $\vec{P}_{z}$, the more the pre-loading bolt is pushed out (in a nondetectable way). It is out of the scope of this design paper, but will be addressed for future version of the fingertip sensor.

\footnotetext{
${ }^{1}$ a set of 0515355582109136163213 grams of hanging weights were used for each location
}

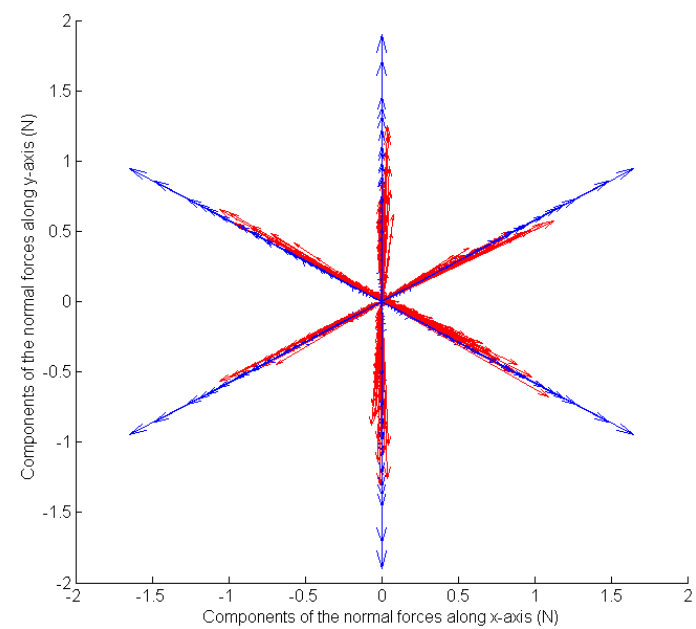

(a) Applied and estimated forces in the $x-y$ plane.

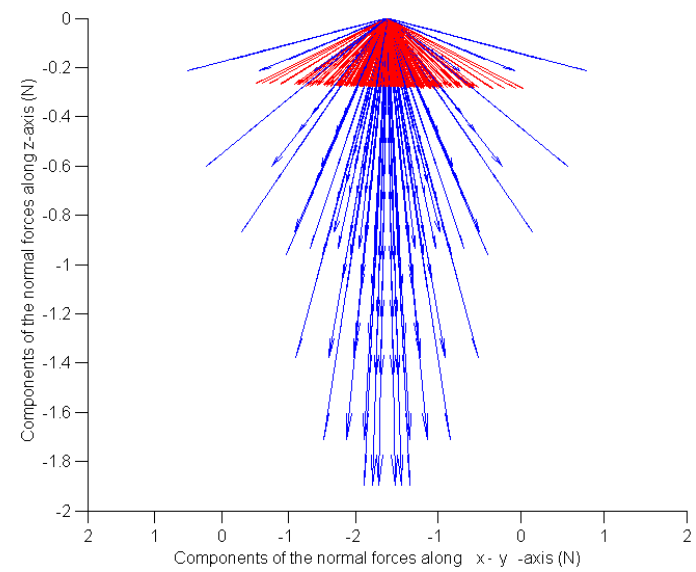

(b) Applied and estimated forces in $\mathrm{z}$-x/y plane.

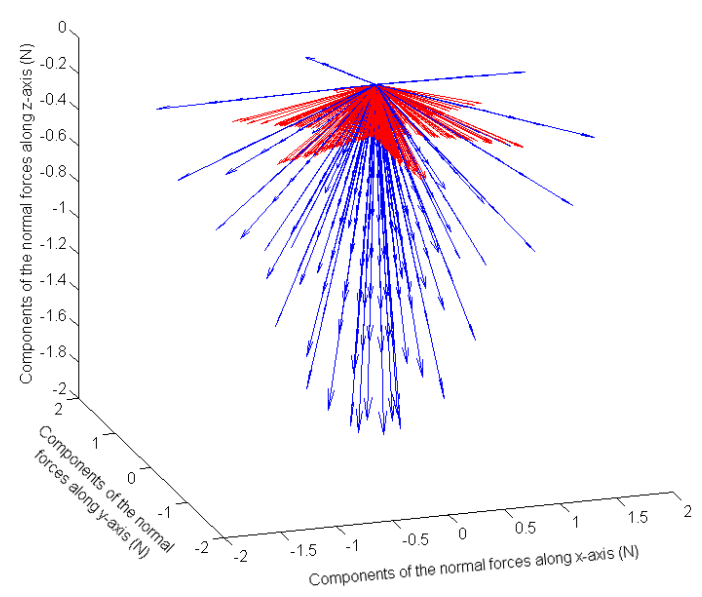

(c) Comparison of the measured and estimated forces in 3D.

Fig. 9. Comparison of applied (blue) and measured (red) normal forces of our proposed force sensor.

\section{A CASE STUDY - DESIGNING FINGERTIP SENSORS FOR PHANTOM ROBOTS' MANIPULATION TASKS}

In this section, our goal is to equip a group of 3-DOF Phantom robots (SensAble Technologies, Inc., Wilmington, MA) with adjustable fingertip sensors so that Phantom robots 


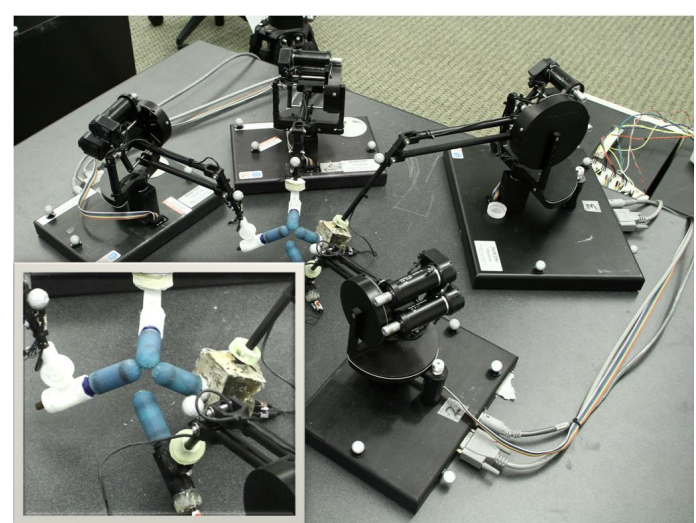

Fig. 10. Fully assembled fingertip sensors mounted at the end-effectors of four 3-DOF Phantom robots for cooperative manipulation tasks.

can perform manipulation tasks cooperatively. The design requirements are listed below:

1) The fingertip sensor needs to be connected to the endeffector of a 3-DOF Phantom robot with a 1-DOF adjustable base.

2) The fingertip used for manipulation objects are expected to be compliant, and can be easily changed to other shapes with little effort.

3) During manipulation tasks, only the radial side of the fingertip sensor is expected to contact with the objects. Therefore the fingertip sensor should be able to detect the directions of the external forces coming sideways. The resolution should be within 60 degrees in the radial plane.

4) The replacement of the fingertip should be easy and fast; the budget of each fingertip should not exceed \$20.

At this stage, the design requirements of the fingertip sensor are all met for the manipulation tasks of the Phantom robots as shown in Fig. 10. More information about the sensor can be found in our video submission. In the rest of this section, we are going to validate the efficacy of our proposed method through experiments.

As illustrated in Fig. 11, a fully assembled fingertip sensor for the Phantom robot was tested in the experiments. A MLP25 load cell (Transducer techniques, CA) was attached to a probe stylus. Infrared markers were used to track the motion of the stylus. The 3D marker coordinates were measured at $480 \mathrm{~Hz}$ using a 7-camera system (PhaseSpace Inc., San Leandro, CA) with respect to the camera (global) frame. The direction of the contact force applied to the fingertip in the radial plane was first calculated in the finger frame and then transformed to global frame for comparison.

The magnitude and the orientation of the contact force were recorded by the the load cell and collected by the motion capture system, respectively. Those values were used to label and validate our experimental results. The FSR sensor changes its resistance based on the force applied to it. We measured the resistance using a voltage divider that divides the $5 \mathrm{~V}$ supply voltage between $10 \mathrm{~K} \Omega$ resistor and the sensor. We then converted the voltage reading to resistance

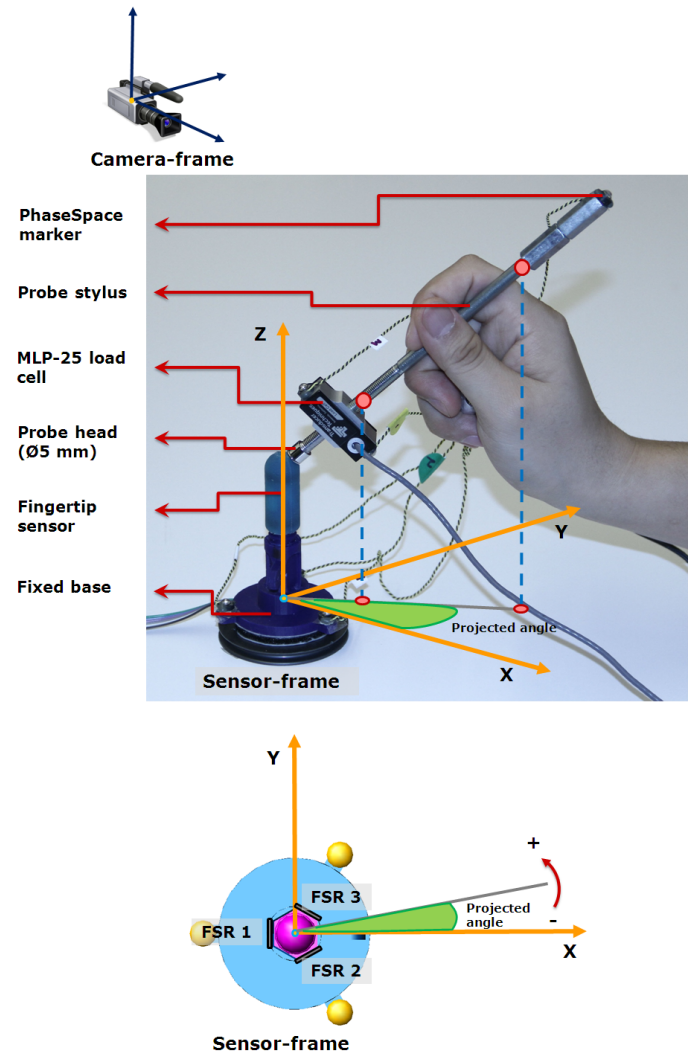

Fig. 11. Experimental setup of the fingertip sensor and definition of different frames. Top: Labeled pictures of a typical trial illustrating the definition of projected angle, sensor-frame, camera-frame, and important components of the experiment. Bottom: Locations of the three FSRs (\$6/unit) in sensor-frame whose $\mathrm{X}-\mathrm{Y}$ plane is the radial plane).

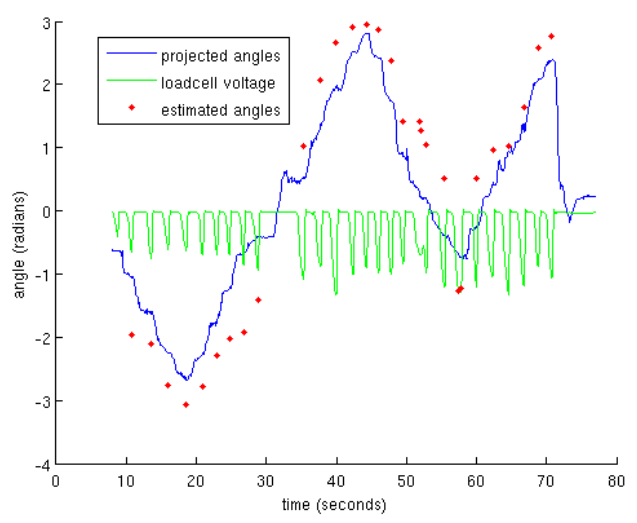

Fig. 12. Comparison of the contact angles between measured and estimated values in the radial plane.

for reporting.

As shown in Fig. 11, the force vectors measured from the probe stylus were first projected onto the $\mathrm{X}-\mathrm{Y}$ plane (the radial plane) in the sensor-frame. Then the projected angles were calculated based on their $\mathrm{X}$ and $\mathrm{Y}$ coordinates. The projected angle is equivalent to the $\beta$ value as modeled in the section IV, and therefore its value can be calculated by using Eq. (3) and (4). 
The comparison result between the measured and the estimated projected angles is shown in Fig. 12. Note that the readings from the load cell are only used as the contactevent indicator. When the contact occurs, a spike would be observed in the load cell data. The differences between measured and estimated angles are all within 60 degrees. This means the fingertip sensor could successfully meet our design requirements for the Phantom robot. The sources of the discrepancies between the measured and estimated angles most likely result from the hysteresis and non-linearity of the FSRs [14]. In addition, the contacts between the probe head and the silicone rubber coated fingertip seemed to be more complicated than we expected - the effect of the deformation of the silicone rubber around the probe head combined with the relatively large friction made it hard to accurately measure the direction of the applied force.

\section{CONCLUSION AND FUTURE WORK}

We have detailed the design of a low-cost, 3-axis fingertip sensor that is made of 3D-printed materials. Our proposed fingertip sensor is composed of a fingertip, a FSS sensor enclosed base, a cantilever-bolt, and a set of low-cost sensors. In order to improve our design concept, experiments were conducted on optimizing the effects of configurations on the performance of the fingertip forces. In total, over 2000 loading/unloading tests were conducted for collecting data used in statistical analysis. We found that there was no obvious effects on the linearity of the sensor by pre-loading the FSS sensors with the cantilever bolt. However the height of the 3D-printed fingertip appears to be an important factor that affects the hysteresis performance of the FSS sensor. Thus, an optimized fingertip sensor was used for the calibration in the later experiments. The results of the calibration prove that our proposed fingertip sensor can detect the normal component of the contact forces. However it was challenging for the fingertip sensor to estimate the forces acting along the axial direction of the fingertip sensor, the discrepancy is likely from the elastic deformation of the bottom cap. In our future work, we are planning to add this effects into the fingertip model.

At the end, a case study was presented to validate the adaptive feature of our design concept. Four Phantom robots were equipped with modified fingertip sensors. And each of them costs less than $\$ 20$ to fabricate. Design requirements such as compliance, resolution, and low-cost were all achieved through combining 3D-printing technology and innovative design. Next we are going to perform manipulation with those Phantom robots.

As for improving the functionality of the 3D-printed sensor itself, we are interested in incorporating three more sensors into the design so that the resulting sensor will have the potential to measure all the six degrees of freedom of a contact force. As we demonstrated in our case-study, similar design concept can be easily adapted to different applications with little effort. Due to its compact size, our optimized fingertip sensor will be implemented into a 20DOF anthropomorphic robotic hand that was previously developed in our lab [9]. Eventually, we would like to make our 3D model of the fingertip sensor an open-source sensor so that more researchers can benefit from the convenience and low-cost of the 3D printing technology.

\section{ACKNOWLEDGEMENTS}

This work was supported by the NSF and DARPA. The authors would like to thank Vikash Kumar for his help on setting up the experiments.

\section{REFERENCES}

[1] R. Dahiya, G. Metta, M. Valle, and G. Sandini, "Tactile sensing from humans to humanoids," IEEE Transactions on Robotics, vol. 26, no. 1, pp. 1-20, 2009.

[2] A. Schmitz, M. Maggiali, L. Natale, B. Bonino, and G. Metta, "A tactile sensor for the fingertips of the humanoid robot icub," in IEEE/RSJ International Conference on Intelligent Robots and Systems (IROS), , 2010, pp. 2212-2217.

[3] N. Wettels and G. Loeb, "Haptic feature extraction from a biomimetic tactile sensor: Force, contact location and curvature," in IEEE International Conference on Robotics and Biomimetics (ROBIO), 2011, pp. 2471-2478.

[4] B. Edin, L. Beccai, L. Ascari, S. Roccella, J.-J. Cabibihan, and M. Carrozza, "Bio-inspired approach for the design and characterization of a tactile sensory system for a cybernetic prosthetic hand," in IEEE International Conference on Robotics and Automation(ICRA), 2006, pp. 1354-1358.

[5] T. B. Martin, R. Ambrose, M. Diftler, J. Platt, R., and M. J. Butzer, "Tactile gloves for autonomous grasping with the nasa/darpa robonaut," in IEEE International Conference on Robotics and Automation (ICRA), vol. 2, 2004,.

[6] K. Hosoda, Y. Tada, and M. Asada, "Anthropomorphic robotic soft fingertip with randomly distributed receptors," Robotics and Autonomous Systems, 2006.

[7] Z. Xu, B. Dellon, and Y. Matsuoka, "Design of artificial skin with integrated tactile sensors for anthropomorphic robotic hands," in 2011 IEEE International Conference on Robotics and Biomimetics (ROBIO), 2011

[8] R. R. Ma, L. U. Odhner, and A. M. Dollar, "A modular,open-source $3 \mathrm{~d}$ printed underactuated hand," in IEEE International Conference on Robotics and Automation(ICRA), 2013.

[9] Z. Xu, V. Kumar, and E. Todorov, "A low-cost and modular, 20DOF anthropomorphic robotic hand: Design, actuation and modeling," in IEEE-RAS International Conference on Humanoid Robots (Humanoids), 2013.

[10] Z. Xu, E. Todorov, B. Dellon, and Y. Matsuoka, "Design and analysis of an artificial finger joint for anthropomorphic robotic hands," in 2011 IEEE International Conference on Robotics and Automation (ICRA), 2011.

[11] B. Winstone, G. Griffiths, C. Melhuish, T. Pipe, and J. Rossiter, "TACTIP-tactile fingertip device, challenges in reduction of size to ready for robot hand integration," in 2012 IEEE International Conference on Robotics and Biomimetics (ROBIO), 2012.

[12] I. Honeywell International, "Honeywell fss015wnsx specification," 2013.

[13] Y.-C. Huang, Y.-H. Chang, and W.-S. Chang, "A new structure for a fingertip force sensor," IEEE/ASME Transactions on Mechatronics, vol. 4, no. 3, pp. 293-300, 1999.

[14] A. Hollinger and M. M. Wanderley, "Evaluation of commercial forcesensing resistors," in NIME06, Paris, France, 2006. 